

BRAZILIAN JOURNAL

$\mathrm{OF}$

RADIATION SCIENCES

02-3A (2014) 01-14

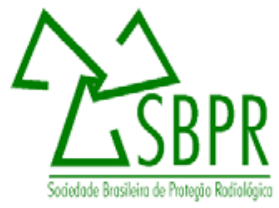

\title{
Evaluacion de incidentes operacionales en el reactor de investigacion rp-10 según escala ines
}

\author{
Rolando W. Arrieta B, Mariano Vela M. \\ Instituto Peruano de Energía Nuclear (IPEN) / Departamento de Operación de Reactores \\ Av. Canadá 1470, Lima 41, Perú \\ rarrieta@ipen.gob.pe, mvela@ipen.gob.pe
}

\begin{abstract}
This report presents the evaluation of the events in 2011 in the RP-10 Nuclear Reactor Nuclear Center Huarangal from the point of view of safety. To classify these events produced is used Scale International Nuclear and Radiological Event Scale (INES) to facilitate a common understanding between the technical community, the media and the general public. From the results we can say that in 2011 all related to security events that occurred in the RP -10 are classified as "below scale" or no safety significance.
\end{abstract}

\section{INTRODUCCION}

En el Reactor Nuclear RP-10 [1], la seguridad ha sido, es y será evaluada en forma permanente [2], actualmente está en su fase de explotación donde es posible generar sucesos relacionados con su seguridad que tienen diversos grados de importancia. 
En el P-10 se realizan un amplio espectro de prácticas [3] que deben ser tratados con diversos criterios de seguridad $[4,5,6]$ y cumplir con regulaciones al respecto [7, 8, 9]. Al margen de todo esto pueden ocurrir sucesos que pueden dañar a las personas y el medio ambiente o afectar las barreras y controles radiológicos o la defensa en profundidad [10]. Todo lo anterior ha servido para que se elabore una escala internacional de clasificación de estos sucesos, de forma que la comunicación de los riesgos radiológicos asociados pueda hacerse de forma coherente en los distintos países, desde el año 2001 [11].

En consecuencia con lo afirmado gestamos este artículo para clasificar los sucesos ocurridos en el RP-10 durante el 2011, desde el punto de vista de la seguridad utilizando la Escala INES [12] a fin de mostrar la importancia que tiene la comunicación abierta de dichos sucesos, de manera que se explique claramente su trascendencia.

La escala no se aplica a todos los sucesos que se han producido en nuestras instalaciones, quedando al margen la seguridad física, o los asociados a la seguridad industrial o con otros sucesos que carezcan de importancia para la seguridad nuclear o radiológica; por ejemplo: peligro únicamente químico, o caída o descarga eléctrica, etc.

\section{MATERIALES Y METODO}

Para este trabajo nos sirve de materiales de estudio los incidentes operacionales y los accidentes que son registrados [13], anualmente, cuyo resumen presentamos en la tabla 1, y estos son tratados según lo normado en la licencia de operación [14].

Para la clasificación de los sucesos se hace uso del Manual del usuario de INES [12] enfocándonos en la secciones 5 y 6 que nos presenta las orientaciones para clasificación de sucesos basados sobre defensa en profundidad. Las orientaciones que se siguen para clasificar por consecuencias reales (secciones 2 y 3): sucesos relacionados con: las personas y el medio ambiente y efecto en las barrearas y controles radiológicos no se aplican a nuestros casos por que no se dieron durante el año de análisis, así como tampoco se tiene en cuenta los incidentes en el transporte.

Tabla 1: Incidentes en el reactor RP-10 durante el año 2011

\begin{tabular}{cccl}
\hline Suceso & $\begin{array}{c}\text { Fecha y } \\
\text { Hora }\end{array}$ & \multicolumn{1}{c}{ Incidente } & Estado del Reactor \\
& $\begin{array}{c}\text { Enero 06 } \\
12: 15\end{array}$ & $\begin{array}{l}\text { SCRAM por desprendimiento de barra } \\
\text { de seguridad 1 }\end{array}$ & $\begin{array}{l}\text { Plena potencia } \\
(10 \mathrm{Mw})\end{array}$ \\
\hline
\end{tabular}




\begin{tabular}{|c|c|c|c|}
\hline 2 & $\begin{array}{l}\text { Marzo } 01 \\
11: 40\end{array}$ & $\begin{array}{l}\text { SCRAM por corte de energia elétrica } \\
\text { externa }\end{array}$ & $\begin{array}{l}\text { Plena potencia } \\
(20 \mathrm{Kw})\end{array}$ \\
\hline 3 & $\begin{array}{l}\text { Marzo } 04 \\
13: 50\end{array}$ & $\begin{array}{l}\text { SCRAM por corte de energia elétrica } \\
\text { externa }\end{array}$ & $\begin{array}{l}\text { Plena potencia } \\
(20 \mathrm{Kw})\end{array}$ \\
\hline 4 & $\begin{array}{l}\text { Abril } 05 \\
12: 53\end{array}$ & $\begin{array}{l}\text { SCRAM por corte de energia elétrica } \\
\text { externa }\end{array}$ & $\begin{array}{l}\text { Plena potencia } \\
(320 \mathrm{Kw})\end{array}$ \\
\hline 5 & $\begin{array}{l}\text { Mayo } 13 \\
13: 42\end{array}$ & $\begin{array}{l}\text { SCRAM por mínimo nivel de cuentas } \\
\text { en arranque disparo de los } 3 \text { LA. }\end{array}$ & En etapa de arranque \\
\hline 6 & $\begin{array}{l}\text { Julio } 31 \\
11: 02\end{array}$ & $\begin{array}{l}\text { SCRAM por disparo NS2X4 y } \\
\text { NS3X4 }\end{array}$ & $\begin{array}{l}\text { En etapa de marcha } \\
(10 \mathrm{Mw})\end{array}$ \\
\hline 7 & $\begin{array}{l}\text { Setiembre } 02 \\
11: 53\end{array}$ & $\begin{array}{l}\text { SCRAM por alto salto de temperatura } \\
\text { en el núcleo disparo de } \Delta \mathrm{TNiY} 4\end{array}$ & $\begin{array}{l}\text { En etapa de marcha } \\
(10 \mathrm{Mw})\end{array}$ \\
\hline 8 & $\begin{array}{l}\text { Octubre } 02 \\
09: 18\end{array}$ & $\begin{array}{l}\text { SCRAM por "alto nivel de tasa de } \\
\text { dosis" en boca de tanque disparo de } \\
\text { LMAiy4 }\end{array}$ & En etapa de arranque \\
\hline 9 & $\begin{array}{l}\text { Octubre } 18 \\
10: 25\end{array}$ & $\begin{array}{l}\text { Se halla discordancia en el Núcleo } 36 \text { - } \\
\text { El EECC NN011 en lugar del NN032 }\end{array}$ & Reactor detenido \\
\hline 10 & $\begin{array}{l}\text { Diciembre } \\
07 \\
10: 47\end{array}$ & $\begin{array}{l}\text { SCRAM por falla en sistema de } \\
\text { reposición (módulo) }\end{array}$ & $\begin{array}{l}\text { Plena potencia } \\
(10 \mathrm{Mw})\end{array}$ \\
\hline
\end{tabular}

En todos estos sucesos se encontró fallas a las que se tuvo que aplicar medidas correctivas a fin de continuar la operación del reactor; las mismas que son comunicadas a la Autoridad Reguladora del IPEN.

Tabla 2: medidas correctivas 2011

\begin{tabular}{|c|c|c|}
\hline Su- & Falla Encontrada & Medidas Correctivas \\
\hline 1 & $\begin{array}{l}\text { Alteración de componentes: módulo } \\
\text { energización electroimanes - disminuye } \\
\text { corriente de energización de BS1 }\end{array}$ & $\begin{array}{l}\text { Cambio de modulo respectivo y poste- } \\
\text { rior reparación del fallado. } \\
\text { Continúa operación }\end{array}$ \\
\hline 2 & $\begin{array}{l}\text { Falla del sistema de alimentación eléc- } \\
\text { trica al C. N. (Caída de Caída de cable } \\
\text { conductor al Centro Nuclear ) }\end{array}$ & $\begin{array}{l}\text { Se da por concluida la operación para } \\
\text { continuar el día siguiente. }\end{array}$ \\
\hline 3 & $\begin{array}{l}\text { Falla del sistema de alimentación eléc- } \\
\text { trica al C. N. (Nueva caída de cable con- } \\
\text { ductor al Centro Nuclear) }\end{array}$ & $\begin{array}{l}\text { Se da por concluida la operación para } \\
\text { continuar el día siguiente. }\end{array}$ \\
\hline 4 & $\begin{array}{l}\text { Falla del sistema de alimentación eléc- } \\
\text { trica al C. N. (Falla recurrente) }\end{array}$ & $\begin{array}{l}\text { Se da por concluida la operación para } \\
\text { continuar el día siguiente. }\end{array}$ \\
\hline
\end{tabular}



No bajar cámaras de arranque para un temporáneo de blancos. movimiento en el núcleo por pedido ex-
Revisión de procedimientos para bajar potencia.
Continúa operación.
Sismo suficiente para accionar sistema
6 de seguridad
Reponer disparos
Continúa operación
Mala fijación de los disparos en los mó-
7 dulos convencionales
Revisión de procedimiento y valores de los disparos.
Continúa operación.
Mala calibración de monitores de área -
realizado en servicio externo
realizado en servicio externo
No se realizó todos los movimientos al
9 configurar el núcleo 32 y se arrastró la
Cambio de monitores de boca de tanque
y su modulo
Continúa operación
Se rectifica la discordancia, se realizan experiencias nuevas y se notifica a misma hasta la el núcleo 36
OTAN
Al reponer una sola cadena, no debía
Pedir a mantenimiento que se respete el
10 producir SRAM, como sucedió ese día.
procedimiento de intervención
Continúa operación

\section{CLASIFICACION DE LOS SUCESOS}

Cada uno de los sucesos de la tabla 1 son complementados con una breve descripción en la tabla 2: fallas encontradas y medidas correctoras.

\subsection{SUCESO 1: Disparo del reactor tras la caída de la barra de seguridad $N^{\circ} 1$ (BS1)}

\subsubsection{Descripción del suceso}

El RP-10 estaba funcionando a 10 MW, con control automático, cuando la BS1 se desprendió de su acople y cayó por gravedad, con esto inhabilita el control automático (al no tener tres barras de seguridad en el límite superior) y no se puede controlar manualmente al no estar habilitado la lógica de secuencia de movimiento [15]. Con esto se propició SCRAM total.

La señal involucrada sirve para tener protección ante un evento inusual en la etapa de arranque, pero en este caso actuó como evento iniciador previsto en el diseño.

\subsubsection{Clasificación del suceso}

Consecuencias reales: No hubo consecuencias reales del suceso.

Frecuencia del iniciador: La caída accidental de barras de seguridad no demanda la actuación de las funciones de seguridad y, por tanto, no es un iniciador. El disparo del reactor es un iniciador (categoría de frecuencia - prevista). 
Operatividad de función de seguridad: La función de seguridad relativa al "control de reactividad” era plena.

Clasificación básica: Hubo un iniciador real. Según [12]- sección 5.1.3, la celda A(1) del cuadro 9 es adecuada y la clasificación básica que da es Debajo de la escala / Nivel 0.

Factores adicionales: Sin motivos para aumentar la clasificación.

Clasificación final: Debajo de la escala / Nivel 0.

\subsection{SUCESO 2: Pérdida de caudal de refrigeración (también aplicadas a sucesos 3 y 4 )}

\subsubsection{Descripción del suceso}

El suceso se produjo por corte de energía eléctrica externa al interrumpirse su suministro por problemas en el sistema de transmisión eléctrico.

Es necesario destacar que el RP-10 tiene una gran piscina de almacenamiento de agua del sistema primario [16]. En caso de pérdida de refrigeración, cualquier calentamiento del agua será extremadamente lento.

\subsubsection{CLASIFICACION}

Consecuencias reales: No hubo consecuencias reales del suceso.

Consecuencias potenciales máximas: Hay que considerar dos funciones de seguridad. Una es la refrigeración del combustible, y la otra es el blindaje para evitar dosis elevadas a los trabajadores. Para ambas funciones de seguridad, por el inventario del RP-10, las consecuencias potenciales máximas no pueden rebasar el nivel 4 por lo que el máximo nivel en función de la defensa en profundidad es el nivel 2.

Determinación del número de barreras de seguridad: Teniendo en cuenta la función de refrigeración, según el diseño las barreras de seguridad son el sistema de intercambio de calor y el gran volumen de agua en el tanque del reactor y la pileta auxiliar piscina, haciendo evidente que la barrera de seguridad principal es el gran volumen de agua que es considerado una barrera de seguridad de gran integridad por:

- El aporte de calor es pequeña para el volumen de agua, de manera que cualquier aumento de temperatura será extremadamente lento. 
- Cualquier reducción del nivel de agua es rápidamente detectada y ese nivel podría reponerse de forma sencilla.

Evaluación de la clasificación básica: Según [12], punto 1, sección 6.2.3.1, la clasificación es debajo de la escala / Nivel 0.

Factores adicionales: Sin motivos para aumentar la clasificación.

Clasificación global: Debajo de la escala / Nivel 0.

3.3 SUCESO 5: Disparo del reactor por mínimo nivel de cuentas en la etapa de arranque tras una operación a baja de potencia

\subsubsection{Descripción del suceso}

Durante el movimiento de las barras para bajar de potencia (luego de estar a $10 \mathrm{MW}$ ) con el fin de poder realizar carga de muestras en posición central: El reactor pasó a la etapa de arranque y se produjo SCRAM debido a una señal de “bajo nivel de cuentas en los canales de arranque”. Esto por que no se bajaron las cámaras de fisión para mejorar el nivel de cuentas [15].

\subsubsection{CLASIFICACION}

Consecuencias reales: No hubo consecuencias reales del suceso.

Frecuencia del iniciador: La señal de bajo nivel de cuentas $<5$ se para proteger contra el fallo de instrumentos, pero en este caso actuó como protección contra fallos de procedimientos. Por esto se tiene un iniciador real de categoría de frecuencia - prevista.

Operatividad de función de seguridad: La función de seguridad relativa al "control de reactividad” era plena. 
Clasificación básica: Al igual que suceso 1, la clasificación básica es debajo de la escala / Nivel 0 .

Factores adicionales: Sin motivos para aumentar la clasificación.

Clasificación final: Debajo de la escala / Nivel 0.

3.4 SUCESO 6: SCRAM por sismo de magnitud mayor a 6 MM - disparo NS2X4 y NS3X4.

\subsubsection{Descripción del suceso}

Luego de estar a potencia, con control automático, se sintió un fuerte sismo que fueron reportados como de magnitud $8 \mathrm{MM}$ en su epicentro. Con este suceso se activó la lógica de seguridad y se produjo SCRAM debido a las señales sísmicas [17] por “nivel alto de sismo”.

\subsubsection{CLASIFICACION}

Consecuencias reales: No hubo consecuencias reales del suceso.

Frecuencia del iniciador: Se produjo un iniciador real, los sismos de gran magnitud. La frecuencia de este iniciador es prevista.

Operatividad de función de seguridad: La función de seguridad relativa al "control de reactividad” era plena.

Clasificación básica: Al igual que sucesos 1 y 5, la clasificación básica es debajo de la escala / Nivel 0.

Factores adicionales: Sin motivos para aumentar la clasificación. 
Clasificación final: Debajo de la escala / Nivel 0.

3.5 SUCESO 7: SCRAM por aparente alto salto de temperatura en el núcleo - disparo de $\Delta$ TNiY4.

\subsubsection{Descripción del suceso}

Se estaba trabajando a la potencia nominal cuando se produjo el disparo de las señales de alto salto de temperatura produciéndose SCRAM, todo esto a pesar de no haber superado el nivel fijado en los límites y condiciones operacionales [17] para el salto de temperatura $\left(7^{\circ} \mathrm{C}\right)$. La causa fue un error en el prefijado de los respectivos comparadores $\left(5^{\circ} \mathrm{C}\right)[18]$.

\subsubsection{CLASIFICACION}

Consecuencias reales: No hubo consecuencias reales del suceso.

Frecuencia del iniciador: Se produjo un iniciador real, los sismos de gran magnitud. La frecuencia de este iniciador es prevista.

Operatividad de función de seguridad: La función de seguridad relativa a la "refrigeración del núcleo” era plena.

Clasificación básica: Al igual que sucesos 1, 5 y 6 la clasificación básica es debajo de la escala / Nivel 0.

Factores adicionales: Sin motivos para aumentar la clasificación.

Clasificación final: Debajo de la escala / Nivel 0. 
3.6 SUCESO 8: SCRAM por aparente “alto nivel de tasa de dosis" en boca de tanque disparo de LMAiy4.

\subsubsection{Descripción del suceso}

El RP-10 estaba en la etapa de arranque - evolucionando para pasar a marcha, cuando se produjo el disparo de las señales de "alto nivel de tasa de dosis en boca de tanque”[17] produciéndose SCRAM, En esta caso si bien la acción de seguridad es la correcta -no hubo fuga de productos de fisión, el error fue haber calibrado mal los tres monitores de área involucrados produciendo falla de causa común al manipular una estación patrón e introducir errores que llegaron a aumentar las lecturas hasta por un orden de magnitud.

\subsubsection{CLASIFICACION}

Consecuencias reales: No hubo consecuencias reales del suceso.

Frecuencia del iniciador: Se produjo un iniciador real, aunque fue sobredimensionado por un error de calibración. La frecuencia de este iniciador es prevista.

Operatividad de función de seguridad: La relativa a “confinamiento de material radiactivo” era plena.

Clasificación básica: Al igual que sucesos 1, 5, 6 y 7, clasificación básica es bebajo de la escala / Nivel 0.

Factores adicionales: Sin motivos para aumentar la clasificación.

Clasificación final: Debajo de la escala / Nivel 0.

3.7 SUCESO 9: Se halla discordancia en el Núcleo 36 - Elemento combustible con código NN011 es hallado en lugar del elemento combustible NN032

IX Latin American IRPA Regional Congress on Radiation Protection and Safety - IRPA 2013

Rio de Janeiro, RJ, Brazil, April 15-19, 2013

SOCIEDADE BRASILEIRA DE PROTEÇÃO RADIOLÓGICA - SBPR 


\subsubsection{Descripción del suceso}

En una inspección rutinaria de salvaguardias se encuentra discordancia entre lo informado a la autoridad regulatoria y el núcleo que estaba configurado [18, 19]. Este error se produjo en el armado de la configuración $\mathrm{n}^{\circ}$ 33, donde no se realizaron todos los movimientos planteados, faltando una permuta entre tres posiciones para optimizar el quemado. Este error fue arrastrado en los núcleos 34, 35 y 36 donde se detectó el error. Se reconfiguró el núcleo, se realizó pruebas de rutina y luego de informar a autoridad regulatoria se continuo con las operaciones.

\subsubsection{CLASIFICACION}

Consecuencias reales: No hubo consecuencias reales del suceso.

Frecuencia del iniciador: No hubo iniciador. Los sistemas de seguridad no fueron requeridos.

Operatividad de la función de seguridad: Las tres funciones de seguridad básicas (control de reactividad, refrigeración del combustible y confinamiento de material radiactivo) no fueron afectados.

Clasificación básica: No hubo iniciador real. Según la sección 5.1.4, la celda A(3) del cuadro 10 es adecuada, y da una clasificación básica que corresponde a: Debajo de la escala / Nivel 0.

Factores adicionales: Sin motivos para aumentar la clasificación.

Clasificación final: Debajo de la escala / Nivel 0.

\subsection{SUCESO 10: SCRAM por falla en sistema de reposición (módulo) o falla de equipo}

\subsubsection{Descripción del suceso}

En plena operación al notar un disparo de la lógica de SCRAM, al apretar el botón de reposición se produce SCRAM total por falla del equipo, al ser esta una acción permitida en cualquier circunstancia.

\subsubsection{CLASIFICACION}

Consecuencias reales: No hubo consecuencias reales del suceso. 
Frecuencia del iniciador: Si bien el fallo de un equipo es un iniciador, por la forma como se produjo el evento, no se tiene un iniciador real.

Operatividad de función de seguridad: Las tres funciones de seguridad básicas (control de reactividad, refrigeración del combustible y confinamiento de material radiactivo) no fueron afectados.

Clasificación básica: No hubo iniciador real. Al igual que caso 9 da una clasificación básica debajo de la escala / Nivel 0 .

Factores adicionales: Sin motivos para aumentar la clasificación.

Clasificación final: Debajo de la escala / Nivel 0.

\section{RESULTADOS}

Los resultados se muestran en cada uno de los 10 casos analizados y en ninguno se tuvieron consecuencias reales que afecten a las personas y/ el medio ambiente o hayan podido impactar en las barreras y los controles radiológicos, por lo cual se evaluó las consecuencias potenciales con los criterios de la defensa en profundidad. En los casos 2, 3 y 4 se dieron el enfoque de consecuencia máxima posible y el número de barreras de seguridad vulneradas, en el resto de casos el enfoque fue desde la perspectiva de suceso iniciador y la degradación de los elementos de seguridad, ambas válidas para nuestro caso por tener información aplicable.

Al margen de los resultados este trabajo es un inicio para implantar un sistema de información coherente con la práctica internacional y servirá de mucho cuando tenga que informarse sucesos de mayor impacto o ser guía para otras instalaciones que quieran utilizar esta escala.

\section{CONCLUSIONES}

Los resultados de los 10 sucesos relacionados con la seguridad del RP-10 son de clasificación final: Nivel 0 que significa como “debajo de la escala” o “sin significado para la seguridad” y esto porque en ningún caso se rebasaron los límites ni las condiciones operacionales - por el correcto funcionamiento de los sistemas de seguridad y/o fueron resueltos de conformidad con procedimientos apropiados. 


\section{AGRADECIMIENTO}

Expresamos nuestro agradecimiento al personal de operación del RP-10 por el registro de los sucesos y la información utilizada en esta publicación.

\section{REFERENCIAS}

1. Instituto Peruano de Energía Nuclear (IPEN), Dirección de Producción/Departamento de Operación: Informe de Seguridad del RP-10, Capítulo I: Introducción y Descripción General, Lima-Perú, Agosto, 1992.

2. Organismo Internacional de Energía Atómica, “Seguridad de las instalaciones nucleares”, Colección Seguridad No 110, OIEA, Viena (1993).

3. Instituto Peruano de Energía Nuclear, Dirección de Producción/Departamento de Operación: Informe de Seguridad del RP-10, Capítulo V: El Reactor RP-10, Lima-Perú, Agosto, 1992.

4. Organismo Internacional de Energía Atómica, "Infraestructura legal y estatal para la seguridad nuclear, radiológica, de los desechos radiactivos y del transporte”, Colección de Normas de Seguridad del OIEA No GS-R-1, OIEA, Viena (2004).

5. Organismo Internacional de Energía Atómica, "Documentation for use in Regulating Nuclear Facilities”, Colección de Normas de Seguridad del OIEA No GS-G-1.4, OIEA, Viena (2002).

6. Organización de las Naciones Unidas para la Agricultura y la Alimentación, Organismo Internacional de Energía Atómica, Organización Internacional del Trabajo, Agencia para la Energía Nuclear de la OCDE, Organización Panamericana de la Salud, Organización Mundial de la Salud, Normas básicas internacionales de seguridad para la protección contra la radiación ionizante y para la seguridad de las fuentes de radiación, Colección Seguridad No 115, OIEA, Viena (1997).

7. Instituto Peruano de Energía Nuclear (IPEN), Oficina Técnica de la Autoridad Nacional: Reglamento de Seguridad Radiológica (D: S: Nº 009-97-EM), Lima, Perú, 1997. 
8. Instituto Peruano de Energía Nuclear (IPEN), Oficina Técnica de la Autoridad Nacional: Reglamento de Protección Física de Instalaciones y Materiales Nucleares (D: S: No 014-2002EM), Lima, Perú, Abril 22 de 2002.

9. Instituto Peruano de Energía Nuclear (IPEN), Oficina Técnica de la Autoridad Nacional: Reglamento de autorizaciones, Fiscalización, Control, Infracciones y Sanciones de la ley 28028 (D: S: No 041-2003-EM), Lima, Perú, Diciembre 11 de 2003.

10. Grupo Internacional Asesor en Seguridad Nuclear, Defensa en Profundidad en Seguridad Nuclear, INSAG-10, OIEA, Viena, 1997.

11. Organismo Internacional de Energía Atómica, Escala Internacional de Sucesos Nucleares (INES), Manual del usuario, Edición de 2001, OIEA, Viena, 2001.

12. Organismo Internacional de Energía Atómica, Escala Internacional de Sucesos Nucleares (INES), Manual del usuario, Edición de 2008, OIEA, Viena, 2010.

13. Instituto Peruano de Energía Nuclear (IPEN), Departamento de Operación, Cuaderno de Incidentes operacionales del RP-10, Lima, Año 2011

14. Instituto Peruano de Energía Nuclear (IPEN), Oficina Técnica de la Autoridad Nacional, Licencia de operación del Reactor Nuclear RP-10, Lima, Perú, Julio 2009.

15. Instituto Peruano de Energía Nuclear (IPEN), Dirección de Producción, Departamento de Operación: Informe de Seguridad del RP-10, Capítulo VIII - Instrumentación y Control, Lima, Perú, Agosto 1992.

16. Instituto Peruano de Energía Nuclear (IPEN), Dirección de Producción, Departamento de Operación, Informe de Seguridad del RP-10, Capítulo VI: Sistema de Refrigeración y Sistemas Conexos, Lima, Perú, Agosto 1992.

17. Instituto Peruano de Energía Nuclear (IPEN), Dirección de Producción, Departamento de Operación, Informe de Seguridad del RP-10, Capítulo XVII: Limites y Condiciones de Operacionales, Lima, Perú, Agosto 1992.

18. Instituto Peruano de Energía Nuclear (IPEN), Oficina Técnica de la Autoridad Nacional (OTAN), Memorándum Nº 399-11-OTAN, Lima, Perú, Octubre 20 de 2011. 
19. Gallardo P. Alberto, “Causas fundamentales y Secundarias que produjeron el error de Configuración del Núcleo 36 del Reactor RP-10 y su impacto en la seguridad de la Instalación”, Informe de Seguridad, Huarangal, Noviembre 2011. 